Campos-Bandrés, I. O. (2021). Actitudes de los maestros en formación hacia las lenguas extranjeras y la educación multilingüe. Revista Electrónica Interuniversitaria de Formación del Profesorado, 24(2), 67-80.

DOI: https://doi.org/10.6018/reifop.428651

\title{
Actitudes de los maestros en formación hacia las lenguas extranjeras y la educación multilingüe
}

\author{
Iris Orosia Campos Bandrés
}

Universidad de Zaragoza

\section{Resumen}

Durante las dos últimas décadas la enseñanza de las lenguas extranjeras ha contado con un gran impulso en la Comunidad Autónoma de Aragón. Sin embargo, todavía está en vías de desarrollo la investigación sobre su situación y resultados tanto a nivel académico como sociolingüístico. El objetivo de este trabajo es explorar las actitudes de los maestros de Educación Primaria en formación en la Universidad de Zaragoza hacia la educación multilingüe y hacia las lenguas extranjeras preponderantes en el sistema educativo autonómico y estatal (inglés y francés), así como conocer el uso que hacen de las mismas. Los resultados del cuestionario completado por 874 alumnos de $1^{\circ}$ y $4^{\circ}$ curso de magisterio muestran un uso moderado de las lenguas extranjeras más allá de las actividades de ocio relacionadas con la música y el cine. También se constata la presencia de actitudes neutras hacia el francés y favorables hacia el inglés y hacia la educación multilingüe, así como el influjo positivo de variables como el perfil lingüístico del sujeto o el curso académico, principalmente significativas en la determinación de las actitudes hacia la educación multilingüe.

\section{Palabras clave}

Actitudes lingüísticas; aprendizaje de lenguas extranjeras; formación del profesorado; multilingüismo.

\section{Contacto:}

Iris Orosia Campos Bandrés, icamposb@unizar.es, Facultad de Ciencias Humanas y de la Educación, C/Valentín Carderera, 4, 22002, Huesca. 


\title{
Teacher training students' attitudes towards foreign languages and multilingual education
}

\begin{abstract}
Foreign languages teaching has been intensely implemented in Aragon during the last two decades. Nevertheless, there are still few researches focused on its situation and results in both the academic and the sociolinguistic area. The main objective of this research is to explore the attitudes of teacher training students in Zaragoza University towards multilingual education and the main foreign languages taught in the educational system regional and national (English and French). The study also aims to examine the use of these languages among future teachers. The research was carried out through a survey with 874 participants from 1st and 4th grade of Primary Education Teacher Training. The results show a moderate use of foreign languages beyond leisure activities related to music and cinema. It is also confirmed the presence of neutral attitudes towards French language and favorable attitudes towards English and multilingual education. It has been found the positive influence of variables like the linguistic profile and the academic grade, mainly sifnificant in the attitudes towards multilingual education.
\end{abstract}

\section{Key words}

Foreign language learning; langauge attitudes; multilingualism; teacher training.

\section{Introducción}

Durante las últimas décadas hemos sido testigos de un incremento a gran escala en el interés hacia el aprendizaje de las lenguas extranjeras. La inquietud social de nuestros días hacia el dominio de los idiomas de uso mayoritario a nivel internacional es tal que algunos autores hablan de una situación sin precedentes ya que, en palabras de Siguán (2005: 199), "en ningún momento de la historia había ocurrido que todos los habitantes jóvenes de un conjunto de países recibiesen una preparación sistemática para poder comunicarse en una lengua extranjera". Dentro de este auge en la promoción y demanda de la enseñanza de estos idiomas destaca el inglés, considerado ya como la lingua franca a consecuencia de su papel dominante en el ámbito económico de los mercados neoliberales occidentales (Moreno Cabrera, 2008), hecho que ha llevado a un buen número de estados a dotar a esta lengua de un importante protagonismo en sus sistemas educativos.

En lo que respecta al estado español fue gracias a la aprobación de la Ley General de Educación, en 1970, cuando se dio el paso a la introducción de la enseñanza de las lenguas extranjeras en la enseñanza obligatoria (Morales et al, 2001), pero su utilización como medio de comunicación en el proceso de enseñanza-aprendizaje de la educación pública comenzó a impulsarse en la década de 1990, a través del proyecto "Secciones Bilingües" (españolfrancés) que se implantó en la etapa de Educación Primaria en Andalucía. Desde entonces, de forma paulatina y con un alto grado de heterogeneidad (vid. Guadamillas y Alcaraz, 2017), las diferentes Comunidades Autónomas iniciaron la implementación de modelos similares en los que el inglés y el francés, principalmente, comenzaron a ganar un mayor protagonismo en las aulas. Así, los datos más recientes, referentes al curso 2018/2019, muestran que en el conjunto del Estado el $84.8 \%$ del alumnado de segundo ciclo de Educación Infantil tiene 
contacto con una lengua extranjera y el $45.4 \%$ de los centros que imparten Educación Primaria y el $45.2 \%$ de los que imparten Educación Secundaria Obligatoria cuentan con proyectos de utilización de un idioma extranjero como lengua de enseñanza, fundamentalmente el inglés, que es el idioma utilizado en el $96,2 \%$ de los programas de aprendizaje integrado de lenguas y contenidos (AICLE)

En el caso de Aragón la implementación del modelo de AICLE empezó a desarrollarse a partir de 1999, cuando el gobierno autonómico asumió las competencias en materia educativa. Así, en ese mismo año se introdujo la enseñanza bilingüe francés-español en ocho centros de Educación Secundaria. También en ese mismo año, cuatro centros aragoneses se sumaron al Programa de Currículo Integrado Español-Inglés que se llevaba desarrollando en otros territorios del Estado desde 1996 en colaboración con el British Council (Liberal, 2013), y mediante el cual el $40 \%$ de las horas del currículo se impartían en inglés. Desde entonces, y en poco menos de dos décadas, la apuesta del Gobierno de Aragón por las lenguas extranjeras se ha hecho patente, sobre todo a partir de 2013, mediante la regulación de estas iniciativas a través de la Orden de 14 de febrero de 2013 por la que se regula el Programa Integral de Bilingüismo en Lenguas Extranjeras de Aragón (PIBLEA) a partir del curso 2013/2014. Desde ese momento, la demanda de este tipo de programas se ha incrementado de forma considerable, de modo que en el curso 2017/2018 un total de 275 centros educativos aragoneses contaron con enseñanzas impartidas a través del inglés (228), francés (43) o alemán (4) (Consejo Escolar de Aragón, 2019), disfrutando el 46.8\% del alumnado de Educación Primaria de una enseñanza basada en el AICLE (Ministerio de Educación y Formación Profesional, 2019). Además, desde 2010, los centros aragoneses de Educación Primaria interesados en ello pueden solicitar la implantación anticipada de la segunda LE en el tercer ciclo de esta etapa, oportunidad que ofrecieron un total de 217 centros en el curso académico 2017/2018 (Consejo Escolar de Aragón, 2019).

Si atendemos a las cifras estatales, reflejadas en la Figura 1, podemos concluir que la Comunidad Autónoma de Aragón se encuentra entre aquellas que encabezan la apuesta por la enseñanza a través de las lenguas extranjeras bajo modelos de aprendizaje integrado de contenidos y lengua extranjera en la etapa de Educación Primaria. Sin embargo, todavía son escasos los datos que conocemos tanto en relación a sus resultados en términos académicos como en cuanto a las actitudes de la comunidad educativa hacia estos idiomas y hacia la enseñanza multilingüe.

Con el propósito de realizar una aportación en este sentido, presentamos una parte de los resultados de una investigación exploratoria bajo el diseño de encuesta centrada en el análisis de los usos y actitudes de los maestros de Educación Primaria en formación en la Universidad de Zaragoza hacia las lenguas de Aragón, las principales lenguas extranjeras presentes en currículo escolar (francés e inglés) y la enseñanza multilingüe.

\footnotetext{
${ }^{1}$ Datos del Ministerio de Educación y Formación Profesional del Gobierno de España, recuperados de: https://www.educacionyfp.gob.es/servicios-al-ciudadano/estadisticas/nouniversitaria/alumnado/lenguas-extranjeras/2018-2019.html.
} 


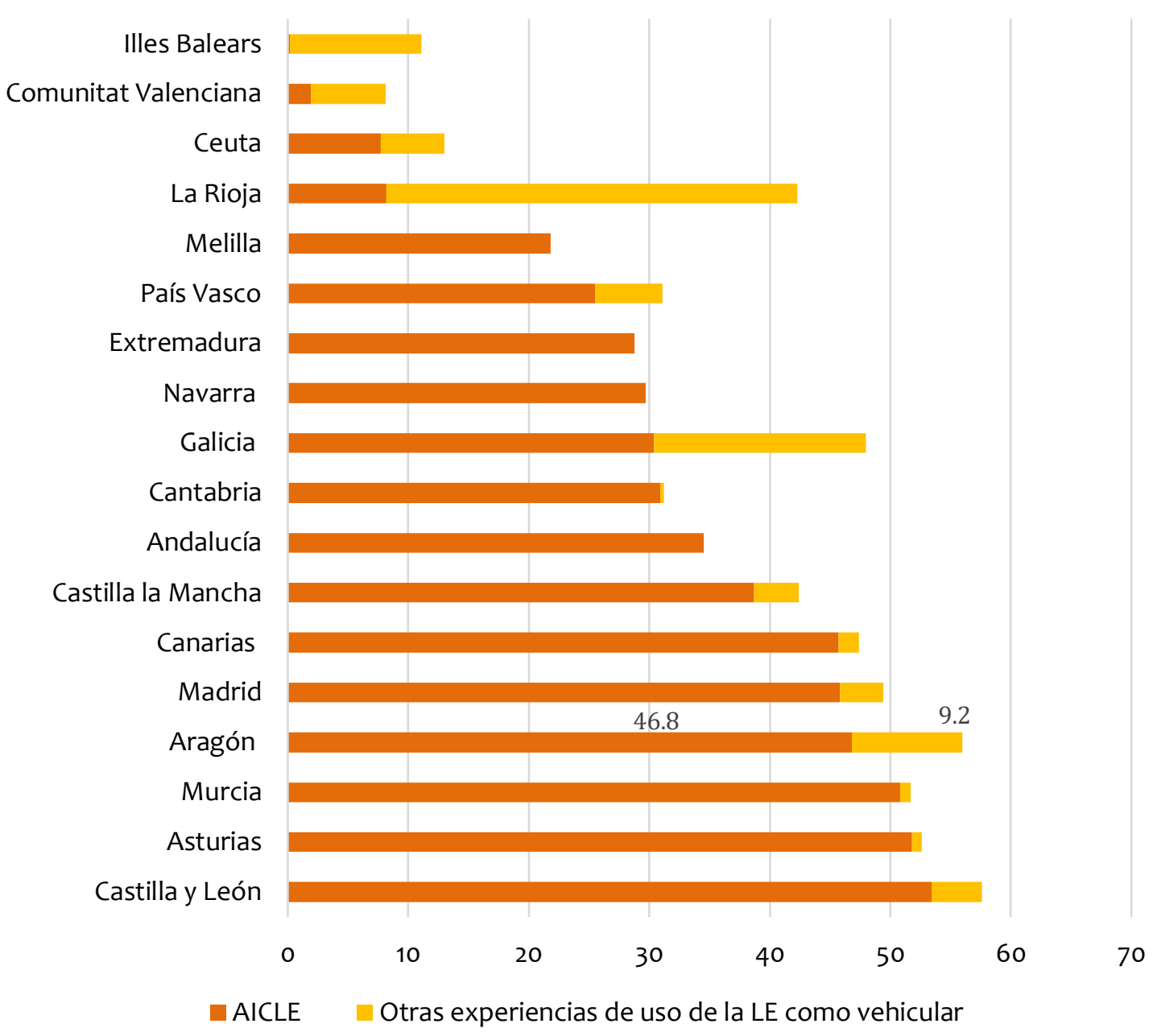

Figura 1. Porcentaje del alumnado de Educación Primaria cursando sus estudios bajo modelo AICLE en cada Comunidad Autónoma. Fuente: Ministerio de Educación y Formación Profesional (2019).

\section{Marco teórico}

Tal y como señalan Bradac, Cargile y Hallet (2001), el estudio de las actitudes lingüísticas ha contado con un desarrollo considerable desde que comenzara a sistematizarse entre las décadas de 1960 y 1970. Este impulso en el estudio científico de las actitudes hacia las lenguas se debió en gran medida a los avances en el ámbito tecnológico, que permitieron el impulso de investigaciones cuantitativas con grandes corpus de datos; así como a la relevancia que la cuestión lingüística comenzaba a tener a mediados del siglo XX en un buen número de estados, centrados tanto en el impulso del conocimiento de las lenguas extranjeras como en la salvaguarda del patrimonio lingüístico propio (Ó Rigain, 2008).

Las primeras investigaciones centradas en el estudio de las actitudes lingüísticas desde una perspectiva psico-social, tanto en términos generales como en lo que respecta al ámbito educativo, se desarrollaron en Canadá, en la década de 1960, con trabajos como el de Lambert, Hodgson, Gardner y Fillebaum (1960), quienes estudiaron las actitudes de los hablantes de inglés y francés de la ciudad de Montreal hacia ambas lenguas desde una perspectiva indirecta, sentando las bases de la técnica más utilizada entre las aproximaciones de tipo indirecto para el estudio de las actitudes lingüísticas, la prueba de matched guise, la cual se centra en la evaluación personal de los hablantes expresándose en las lenguas objeto 
de estudio. El estudio de las actitudes lingüísticas con amplias muestras se generalizó a partir de entonces a otros contextos plurilingües dentro del panorama internacional como Irlanda (Ó Rigáin, 1997) o el País de Gales (Sharp et al, 1973).

Desde la Sociolingüística podemos decir que el concepto de actitud lingüística hace referencia, en términos generales, a una manifestación caracterizada por "centrarse y referirse específicamente tanto a la lengua como al empleo que de ella se hace en sociedad" (Moreno Fernández, 1998: 179), aunque todavía no se ha llegado a un consenso definitivo en la definición de este constructo (Lasagabaster, 2003).

Dentro de la investigación sobre las actitudes lingüísticas ha destacado la desarrollada en el ámbito educativo ya que, tal y como señalan Huguet y González Riaño (2004: 30), "el contexto escolar constituye por sus especiales características de complejidad sociológica, un espacio privilegiado para que tanto actitudes como prejuicios se hagan nítidamente visibles". Asimismo, dentro del estudio de las actitudes hacia las lenguas en el ámbito educativo, ha existido un especial interés por el conocimiento de las valoraciones del profesorado debido al constatado influjo de este colectivo en la formación y cambio de las actitudes del alumnado, ya que los docentes son referentes básicos para el niño en su proceso de socialización (Lasagabaster, 2003). De hecho, en coherencia con esta esta capacidad de potencial influencia, el Marco Europeo Común de Referencia para las Lenguas (Consejo de Europa, 2001), hace hincapié en el papel del profesorado de lenguas en lo que respecta a la consecución de los objetivos europeos en materia de integración y de desarrollo de la competencia pluricultural del alumnado.

En cuanto a los factores que determinan la orientación de las actitudes hacia las lenguas, desde el marco teórico desarrollado hasta nuestros días se ha destacado el influjo de una serie de variables tanto de naturaleza individual como social, como son: la edad, el estatus socioeconómico familiar, la condición lingüística familiar, el contexto sociolingüístico, la motivación asociada al aprendizaje de la lengua en cuestión (instrumental o integradora), el contacto con la misma a través del sistema educativo o el perfil lingüístico del sujeto (monolingüe o bi/plurilingüe). Asimismo, se ha subrayado el peso que los prejuicios hacia el grupo o sociedad hablante de una lengua puede tener en la muestra de unas actitudes negativas hacia la lengua per se (Janés, 2006).

\section{Metodología}

\section{Preguntas y objetivos de investigación}

En nuestra investigación, tomando como referencia el trabajo desarrollado por Huguet (2006) con alumnado de Educación Secundaria Obligatoria, estudiamos las actitudes hacia las principales lenguas de currículo (castellano, francés e inglés) y hacia las lenguas propias de Aragón (aragonés y catalán), completando nuestra herramienta con un apartado destinado al conocimiento de las actitudes hacia la educación multilingüe. Los objetivos de nuestro estudio en lo relativo a las lenguas extranjeras (inglés y francés) fue conocer la orientación de las actitudes que los maestros en formación tienen hacia estas -explorando, además, los factores que pueden determinarlas-, y conocer el uso que hacen de estos idiomas en el ámbito personal y académico.

Nuestras preguntas de investigación fueron:

1) ¿Cuál es la orientación de las actitudes de los maestros aragoneses en formación hacia las lenguas extranjeras y hacia la educación multilingüe? 
2) ¿Qué uso hacen de estas lenguas los futuros maestros en su vida cotidiana, tanto personal como académica?

3) ¿Qué factores (entre los destacados en la literatura previa) determinan el desarrollo de unas actitudes más favorables hacia las lenguas extranjeras y hacia la educación multilingüe?

\section{Participantes}

El estudio se realizó entre el alumnado matriculado en los cursos de $1^{\circ}$ y $4^{\circ}$ del Grado en Magisterio en Educación Primaria en los tres campus de la Universidad de Zaragoza (Huesca, Zaragoza y Teruel). Se contó con un total de 874 participantes, repartidos tal y como queda recogido en la Tabla 1, garantizándose la representación del alumnado que cursaba magisterio en dicha universidad, ya que aplicando la fórmula donde $\mathrm{N}$ es el total de la población o universo, $\mathrm{k}$ la constante que depende del nivel de confianza asignado (en nuestro caso de $95 \%$, por lo que $\mathrm{k}=1,96$ ) y e el error muestral deseado (en nuestro caso del $5 \%$ ) la muestra necesaria para garantizar dicha representatividad era de 317 participantes.

Tabla 1.

Muestra necesaria para garantizar la representatividad y muestra participante.

\begin{tabular}{ccccc}
\hline Campus & $\begin{array}{c}\text { Total alumnado } \\
\text { matriculado }\end{array}$ & $\begin{array}{c}\text { Muestra } 1^{\circ} \text { curso } \\
\text { participante }\end{array}$ & $\begin{array}{c}\text { Muestra } 4^{\circ} \text { curso } \\
\text { participante }\end{array}$ & $\begin{array}{c}\text { Total alumnado } \\
\text { participante }\end{array}$ \\
\hline Huesca & 442 & 153 & 66 & 219 \\
\hline Zaragoza & 968 & 278 & 236 & 514 \\
\hline Teruel & 381 & 80 & 61 & 141 \\
\hline Unizar & 1791 & & & 874 \\
\hline
\end{tabular}

\section{Instrumento}

El cuestionario aplicado fue una adaptación del utilizado por Huguet (2006) entre el alumnado aragonés de Educación Secundaria Obligatoria el cual, a su vez, se ha utilizado en múltiples investigaciones similares desde su creación por Sharp et al. (1973). A este instrumento se añadió un apartado relacionado con la enseñanza multilingüe, de modo que finalmente constó de los siguientes bloques: 1) información personal del estudiante (variables: curso, procedencia, condición lingüística familiar, situación socioprofesional familiar, usos lingüísticos, contacto con el aragonés y/o el catalán a través de su enseñanza); 2) actitudes hacia cada una de las lenguas del currículo aragonés de Educación Primaria -la oficial, las extranjeras y las propias de Aragón- (aragonés, castellano, catalán, francés e inglés) y 3) actitudes hacia la enseñanza multilingüe.

La herramienta fue validada en un estudio piloto (Campos, 2013) que permitió corroborar su consistencia interna con un resultado del Alfa de Cronbach de .864 .

\section{Procedimiento}

En cuanto a la codificación de las respuestas del alumnado respecto sobre cada una de las lenguas del cuestionario, dado que la herramienta era de respuesta dicotómica, se asignó el valor 0 a las respuestas desfavorables $y+1$ a las favorables, de modo que se obtuvo la media de cada sujeto para cada lengua una vez agrupadas las respuestas de los 10 ítems asignados a cada una de ellas. Esta variable cuantitativa se transformó en cualitativa tomando como 
referencia estudios previos en los que se ha utilizado la herramienta seleccionada (González Riaño y Armesto 2004, González Riaño y Huguet 2002, Huguet, 2006): actitud favorable: 7,51 a 10; actitud neutra: 2,51 a 7,50 y actitud desfavorable: 0 a 2,50.

El tratamiento estadístico de los datos se realizó con la ayuda del software SPSS en su versión 15 para Windows. En concreto, se realizaron pruebas estadísticas descriptivas para conocer las tendencias de uso de las lenguas y se utilizó el estadístico ANOVA2 y las pruebas post hoc de Sidak y Games-Howell para analizar el influjo de las variables independientes en las actitudes de los sujetos, así como para determinar entre qué grupos se encontraban las diferencias. El nivel de significación fue de .05.

\section{Resultados}

En lo que respecta a las actitudes mostradas en términos generales hacia las diferentes lenguas (reflejadas en la Tabla 2), encontramos tres grupos actitudinales evidentes. En primer lugar, se reportan actitudes positivas hacia el castellano $(M=7,54$; DT=1,17), primera lengua de la mayor parte de los participantes (el $90 \%$ la utiliza de forma exclusiva en sus relaciones familiares) y hablada por la totalidad de la muestra. También en este primer grupo caracterizado por las actitudes favorables, encontramos al idioma inglés $(M=7,91 ; D T=1,46)$, que obtiene una puntuación incluso superior, aunque sin diferencias estadísticamente significativas, a la de la lengua materna de la mayor parte de los participantes.

En segundo lugar, encontramos las actitudes mostradas hacia una de las lenguas propias de Aragón, el aragonés ( $M=6,23 ; \mathrm{DT}=2,69)$, acompañadas de las actitudes hacia la que ocupa el lugar de segunda lengua extranjera en la mayor parte de los centros educativos aragoneses, el francés $(M=5,74 ; D T=2,05)$. Las actitudes hacia estas dos se encuentran claramente en un rango neutral, si bien resulta llamativo que sean más favorables en el caso de la lengua aragonesa, idioma en estado de minorización lingüística (vid. López, 2013), con un lugar anecdótico en el ámbito escolar restringido a la provincia de Huesca y que, en coherencia con dicho estado de minorización, únicamente ocho sujetos manifiestan haber estudiado y cuatro utilizar habitualmente en el seno familiar (a pesar de que 154, el 17,6\% de la muestra, considera que es la lengua propia de su localidad de procedencia).

Tabla 2.

Medias generales para cada una de las lenguas y para la educación multilingüe.

\begin{tabular}{ccccccc}
\hline & Castellano & Aragonés & Catalán & Inglés & Francés & E. Multilingüe \\
\hline Media & 7,54 & 6,23 & 3,46 & 7,91 & 5,74 & 7,57 \\
\hline N & 799 & 786 & 820 & 831 & 835 & 848 \\
\hline DT & 1,17 & 2,69 & 2,79 & 1,46 & 2,05 & 1,76 \\
\hline
\end{tabular}

Finalmente, y aunque también se situarían dentro del rango neutral, encontramos un tercer bloque formado por las actitudes hacia la lengua catalana $(M=3,46$; $D T=2,79)$, las cuales se encuentran claramente cercanas al rango de las actitudes desfavorables y con una puntuación media que destaca llamativamente respecto al resto de lenguas objeto de estudio. Asimismo, las actitues hacia esta lengua presentan la mayor desviación típica (DT= 2,79 ), lo cual evidencia que es la lengua ante la que se muestran las actitudes más extremas,

\footnotetext{
${ }^{2}$ La homogeneidad de las varianzas se comprobó mediante la aplicación de la prueba de Levene.
} 
seguida del aragonés (DT= 2,69), con una desviación típica muy similar, y del francés (DT= 2,05).

En cuanto a las actitudes hacia la educación multilingüe, encontramos un clima actitudinal de orientación favorable $(M=7,57$; $D T=1,76)$.

Respecto a los usos lingüísticos, cabe destacar la condición lingüística familiar monolingüe de la mayor parte de los participantes. Así, el 90\% de la muestra manifiesta utilizar únicamente el castellano en sus relaciones familiares, ocupando el catalán el segundo lugar, con un $8,1 \%$ que manifiesta utilizar esta lengua de forma predominante y/o junto al castellano. La tendencia es similar en lo que respecta a la comunicación con las amistades, de modo que el castellano sigue siendo la lengua de empleo exclusivo en el 90\% de la muestra en este tipo de relaciones, seguida del catalán, idioma que manifiestan utilizar -en algunos casos junto al castellano- el 7,4\% de los participantes.

En cuanto a las lenguas utilizadas en las actividades de ocio, de nuevo el castellano es la lengua más utilizada, pues un $74,1 \%$ de participantes que manifiesta leer únicamente en este idioma. En lo que respecta a las lenguas extranjeras, el 18,9\% manifiesta realizar lecturas por placer en una lengua extranjera (siempre el inglés y/o el francés) aunque siempre acompañadas del castellano. Finalmente, encontramos un 6,4\% dice leer en castellano y en catalán en sus tiempos de ocio.

La tendencia en lo que respecta al uso de las lenguas extranjeras varía de forma significativa cuando se trata de la lengua en la que se escucha música y se consumen programas de televisión, series o películas. En este caso, el porcentaje de alumnos que únicamente usa el castellano en estas activiades es mucho menor $(26,2 \%)$ y se invierte la tendencia respecto a lo encontrado en el caso de la lectura, pues la mayor parte de la muestra $(66,9 \%)$ manifiesta utilizar el castellano junto a lenguas extranjeras (fundamentalmente el inglés).

En cuanto a las lenguas utilizadas en la comunicación a través de las redes sociales e Internet, de nuevo el castellano es la lengua preponderante, utilizada de forma exclusiva por el $80,7 \%$. Las lenguas extranjeras (de nuevo, prácticamente siempre el inglés y/o el francés) son utilizadas en el $11 \%$ de los casos y siempre junto al castellano.

Finalmente, en relación a los usos lingüísticos dentro del ámbito académico encontramos nuevamente una discreta utilización de las lenguas extranjeras. Así, para la realización de lecturas vinculadas a sus estudios universitarios, el porcentaje de alumnos que solo utiliza el castellano asciende hasta el $78,7 \%$, seguido de un 19,3\% que, además del castellano, utiliza lenguas extranjeras (siempre el inglés y/o el francés). Sucede algo similar en cuanto a la lengua en la que los maestros en formación se relacionan con sus profesores. En el $88 \%$ de los casos la comunicación es exclusivamente en castellano y un 11,9\% dice utilizar, además, el inglés o el francés. 


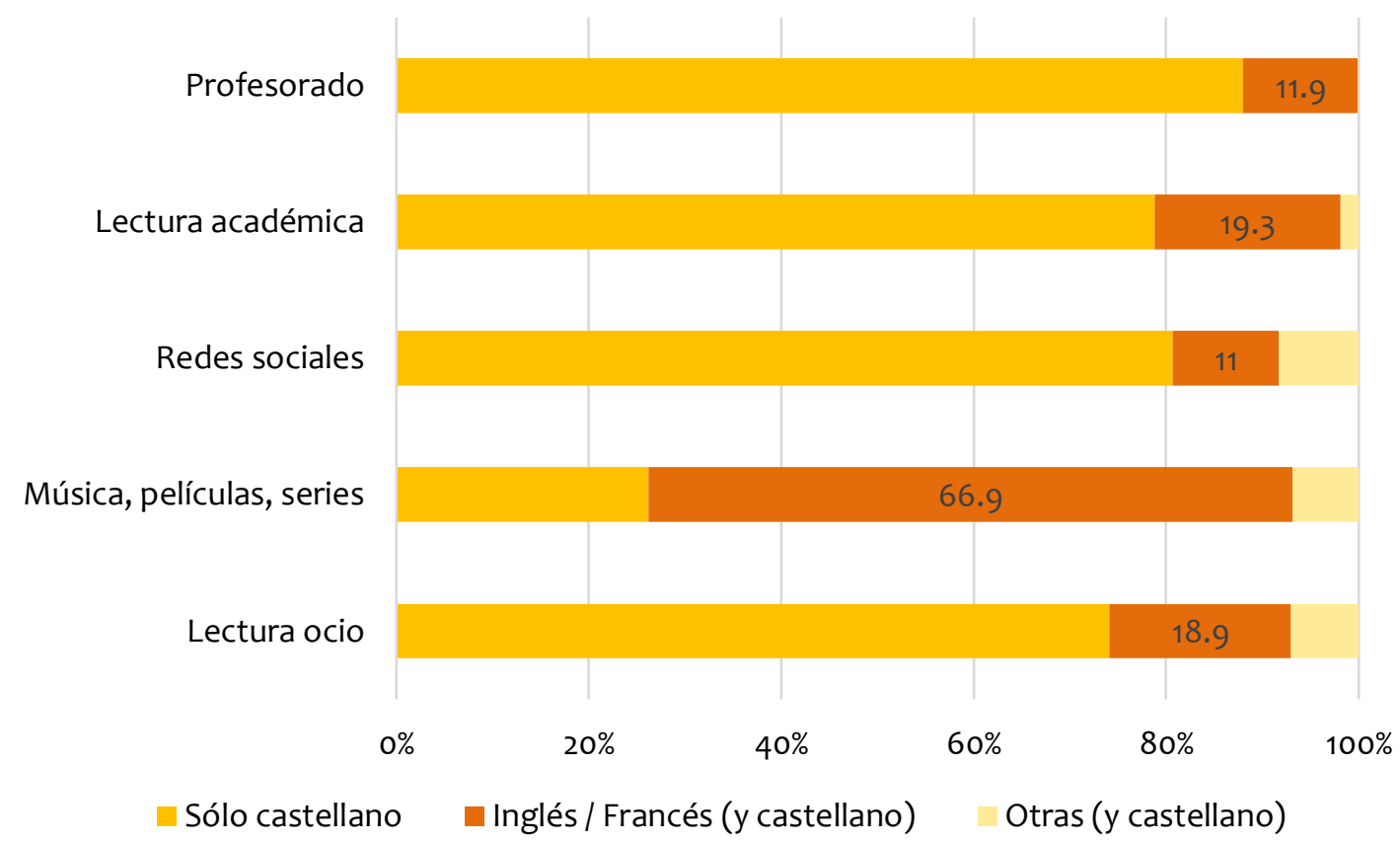

Figura 2. Distribución de la muestra (porcentajes) en función de las lenguas utilizadas en las actividades de ocio y en las actividades académicas.

En cuanto a los factores que reportan diferencias significativas en las actitudes hacia el inglés, la primera variable que destaca es el nivel socioeconómico familiar del alumnado, y en concreto la variable referente al nivel de estudios del padre $(F=2,865 ; p=.009)$. En este sentido, la prueba post hoc de Sidak reporta diferencias significativas $(p=.005)$ entre aquellos sujetos cuyo progenitor cuenta con el graduado escolar $(M=7,57$; $D T=1,60)$ y aquellos que manifiestan que tiene estudios universitarios $(M=8,01$; DT=1,47), aunque en ambos casos las actitudes son favorables.

Otras variables que reportan diferencias significativas en las actitudes hacia el inglés son aquellas relacionadas con los usos lingüísticos en las actividades de ocio. En términos generales, encontramos una relación entre la muestra de actitudes más favorables hacia esta lengua y la manifestación de su uso en la lectura por placer, la escucha de música, el visionado de documentos audiovisuales y la comunicación a través de las redes sociales. En primer lugar, se encuentran diferencias $(F=3,832 ; \mathrm{p}=.010)$ en relación a las lenguas utilizadas para la lectura por placer. Dado que no existe igualdad de las varianzas entre los grupos (Levene= 11.440; $p=.000$ ), tras aplicar el estadístico de Games Howell se encuentran diferencias entre aquellos que utilizan lenguas extranjeras (siempre inglés y/o francés) en este tipo de lectura y aquellos que utilizan de forma exclusiva el castellano $(p=.001)$. Obviamente, las actitudes hacia el inglés son más favorables entre aquellos que utilizan las lenguas extranjeras en la lectura por placer $(M=8,26$; DT = 1,16), aunque en el caso de los que leen exclusivamente en castellano las actitudes hacia el inglés también se encuentran en el rango de favorables $(M=$ 7,84; DT=1,45). Sucede lo mismo cuando tomamos como variable independiente el uso de las lenguas extranjeras para escuchar música o visionar películas y series. En este caso, de nuevo encontramos diferencias significativas $(F=5,270 ; p=.005)$ y la prueba de Games Howell (Levene $=10,255 ; p=.000)$ apunta a que las diferencias $(p=.001)$ se encuentran entre los participantes que manifiestan utilizar de forma exclusiva el castellano en estas actividades y aquellos que utilizan también las lenguas extranjeras. De nuevo, la media es superior en el segundo grupo $(M=8,09$; DT=1,30, frente a $M=7,63$; DT=1,59). 
Finalmente, encontramos también diferencias al tomar como variable independiente los usos lingüísticos en la comunicación a través de las redes sociales e Internet $(F=3,542 ; p=.014)$. Como en los casos anteriores, las diferencias se encuentran entre el alumnado que únicamente utiliza el castellano y aquel que, junto a esta lengua, utiliza algún idioma extranjero $(p=.006)$. Como en los casos anteriores, las actitudes son más favorables en el caso de los participantes que utilizan lenguas extranjeras (siempre inglés y/o francés), además del castellano, en estas situaciones comunicativas $(M=8,33$; DT= 1,14 frente a $M=$ 7,88; DT= 1,43).

En sínstesis, a pesar de las diferencias encontradas, todos los grupos comparados muestran actitudes favorables hacia el inglés, lo que parece evidenciar la consolidación de este idioma como lingua franca.

En el caso del francés los resultados, en términos generales, se asemejan a los encontrados para el caso del inglés, con un escaso número de variables que reportan diferencias significativas, si bien existen algunos datos llamativos. En primer lugar, encontramos el influjo del contexto sociolingüístico $(\mathrm{F}=11,317 ; \mathrm{p}=.000)$, a través de la consideración subjetiva de la existencia (o no) de una lengua propia en la localidad de procedencia. En este sentido, la aplicación de la prueba de Games Howell (Levene $=4,915 ; p=.008$ ) muestra que aquellos participantes que consideran que la lengua aragonesa es una de las lenguas propias de su localidad de procedencia presentan actitudes significativamente diferentes $(p=.000)$ a las de aquellos que consideran que no existe ninguna lengua propia en la localidad de procedencia, siendo superior la media en el caso de los que consideran que el aragonés es una lengua propia de su localidad $(\mathrm{N}=145 ; M=6,35 ; \mathrm{DT}=1,92)$ frente a quienes manifiestan que en su localidad de procedencia únicamente se habla el castellano ( $N=609 ; M=5,56 ; \mathrm{DT}=2,09)$.

Otra variable que reporta diferencias estadísticamente significativas es el sexo del sujeto $(\mathrm{F}=$ 28,069; $\mathrm{p}=.000$ ), de modo que las mujeres presentan actitudes más favorables hacia esta lengua $(M=5,97$; DT= 1,96) que los hombres $(M=5,08 ; \mathrm{DT}=2,18)$.

Es en relación a las actitudes hacia la educación multilingüe donde se encuentra la actuación de una mayor cantidad de las variables contempladas en el estudio. En primer lugar, cabe destacar la presencia de diferencias $(F=15,905 ; p=.000)$ en función de si el sujeto ha estudiado una de las lenguas propias de Aragón (aragonés o catalán). La aplicación de la prueba de Sidak (Levene $=1,095 ; p=.124)$ muestra que las diferencias $(p=.000)$ se encuentran entre los que no han estudiado ninguna de las lenguas propias de Aragón $(M=7,45 ; D T=1,76)$ y aquellos que han estudiado catalán $(M=8,55$; DT $=1,58)$, siendo en el caso de las primeras actitudes neutrales -aunque prácticamente ya en el rango de favorables- y en el de los últimos actitudes claramente favorables. En la misma línea, se encuentran diferencias significativas en función de la condición lingüística familiar ( $F=4,960 ; p=.002)$, de modo que la prueba de $\operatorname{Sidak}$ (Levene $=1,004 ; p=.390$ ) determina que las diferencias se encuentran entre el alumnado monolingüe castellano y aquel que utiliza el catalán en el seno familiar ( $p=.008)$. En coherencia con lo anterior, las actitudes son más favorables entre el alumnado con una condición lingüística familiar catalanoparlante $(M=8,21 ; D T=1,69$, frente a $M=7,51 ; D T=1,76)$. La tendencia se corrobora si tomamos como variable independiente la lengua utilizada con los amigos $(F=6,639 ; p=.000)$, de modo que, según reporta la prueba post hoc de Sidak (Levene $=1,461 ; p=.224$ ), aquellos que únicamente se comunican en castellano presentan unas actitudes significativamente diferentes $(p=.001)$ que aquellos que también utilizan el catalán, siendo de nuevo más favorables las actitudes hacia el multilingüismo entre este segundo grupo $(M=8,35 ; \mathrm{DT}=1,63$, frente a $M=7,49 ; \mathrm{DT}=1,75)$ y situándose quienes tienen un perfil monolingüe en el rango de actitudes neutras hacia la educación multilingüe -si bien con una clara tendencia favorable-. 
Otra variable que reporta diferencias significativas $(F=3,992 ; \mathrm{p}=.046)$ es el curso en el que se encuentran los participantes, de modo que aquellos que se encuentran finalizando sus estudios de magisterio prsesentan unas actitudes más favorables hacia la educación multilingüe $(M=7,72 ; \mathrm{DT}=1,74)$ que los que acaban de comenzar su formación como maestros $(M=7,47 ; \mathrm{DT}=1,77)$, mostrando los primeros unas actitudes favorables y los segundos neutras.

Finalmente, se encuentran diferencias en función del sexo $(F=13,282 ; p=.000)$ con unas actitudes más favorables hacia la educación multilingüe entre las mujeres $(M=7,70 ; D T=1,66)$ que entre los hombres $(M=7,20 ; D T=1,99)$, siendo las de ellas favorables y las de ellos neutras.

\section{Discusión y conclusiones}

En primer lugar, podemos concluir que las actitudes hacia las lenguas extranjeras de mayor peso en el currículo aragonés de Educación Primaria son favorables para el caso del inglés y neutras para el del francés, a pesar de que el uso que los futuros docentes hacen de estos idiomas es bastante limitado tanto en el ámbito personal y de ocio como en el académico, con la excepción del consumo de música, películas y series, actividades en las que más de la mitad de la muestra manifiesta utilizar con asiduidad el inglés y/o el francés, aunque fundamentalmente el primero. En este sentido, parece existir una paradoja en el caso del inglés ya que se demuestran muy favorables hacia el idioma pero existe un escaso uso real. Esta realidad, que también se ha constatado en trabajos recientes (Herrera y Pozas, 2020), podría explicarse por una motivación mayoritaria hacia su aprendizaje de naturaleza instrumental y no tanto impulsada por el deseo de acercarse a las culturas y sociedades anglófonas. En este sentido, investigaciones previas realizadas con alumnado universitario (Lasagabaster, 2004) han evidenciado que la presencia de una motivación de aprendizaje del inglés fundamentalmente instrumental se traduce en el desarrollo de actitudes favorables hacia el idioma que no se corresponde con una competencia comunicativa elevada. En una misma línea, el hecho de que existan diferencias en las actitudes hacia el inglés entre aquellos estudiantes que incorporan esta lengua en actividades de ocio como la lectura por placer o el consumo de música y películas corroboraría el peso que la motivación de naturaleza integradora -relacionada con el deseo de profundizar en la cultura que da sentido al idioma y/o con formar parte de la sociedad que lo habla (Gardner, 1985)- tiene en la determinación de las actitudes lingüísticas.

Por otra parte, cabe destacar la escasa significatividad de la mayor parte de las variables consideradas en la orientación de las actitudes hacia las lenguas extranjeras -y sobre todo hacia el inglés-, lo cual dista de la situación encontrada respecto a las lenguas propias de Aragón (Campos, 2014; Campos, 2015). En el caso del inglés, considerando la orientación favorable de las actitudes y el escaso efecto de las variables independientes, la situación encontrada pordría considerarse como una evidencia de la consolidación de este idioma como lingua franca en el ideario colectivo de los futuros docentes, corroborando así los resultados de investigaciones como las de Herrera y Pozas (2020) o Marín, Alzás y Villares (2020). Así, los resultados parecen evidenciar el impacto tanto del impulso y promoción del estudio de esta lengua que se ha implementado desde las instituciones estatales y autonómicas durante las últimas décadas, como de su valor instrumental en el plano económico (Moreno Cabrera, 2008). En este sentido cabe destacar que en el caso de Aragón se ha constatado recientemente la evolución en positivo de las actitudes hacia el inglés entre la población adolescente (Campos, 2021), pasando de situarse en el rango de neutrales en 2006 al de favorables en la actualidad, lo que constata el impacto positivo del impulso de los programas CLIL en Educación Primaria y Secundaria durante los últimos años. 
Ya por lo que respecta a la diferencia en la orientación de las actitudes hacia las dos lenguas (inglés: actitudes favorables y francés: actitudes neutras) esta podría venir explicada por la relación que se ha demostrado entre el contacto con una L2/L3 a través del sistema educativo y la manifestación de actitudes favorables hacia la misma. Así, a pesar de que el vínculo existente entre las dos variables todavía no es claro en términos de causalidad y efecto, investigaciones desarrolladas en múltiples escenarios lingüísticos han evidenciado dicha relación (Lasagabaster y Huguet, 2007). En este sentido, cabe recordar que en el caso del alumnado participante en nuestra investigación la lengua de estudio obligatorio a lo largo de toda su vida académica ha sido el inglés, ocupando el francés un lugar de optatividad a partir de la etapa de Educación Secundaria en la mayor parte de los casos, por lo que el mayor contacto con la lengua inglesa a lo largo de la trayectoria académica de los participantes podría explicar esa mejor valoración en comparación con el francés. En cualquier caso, como propuesta de mejora y para poder llegar a conclusiones sólidas al respecto, hubiera sido oportuno contar en nuestro instrumento con una pregunta relacionada con el número de años en los que el sujeto cursó francés como segunda lengua extranjera y con otra pregunta relacionada con su posible experiencia cursando un programa de educación bilingüe castellano-francés, dado que en el momento en el que las cohortes participantes en nuestra investigación cursaron las etapas de Educación Primaria y Secundaria estas experiencias ya se estaban comenzando a desarrollar en Aragón. La incorporación de estas dos cuestiones nos habría permitido conocer si un mayor (o más prolongado) contacto con el francés a través del ámbito académico es una variable significativa en la orientación de las actitudes de los futuros docentes hacia esta lengua, hecho que nos ayudaría a explicar también la amplitud de la desviación típica encontrada en los resultados respecto a esta lengua.

Es en las actitudes hacia la educación multilingüe, que en términos generales son favorables, donde se aprecia el influjo de un mayor número de variables. Entre ellas destaca el perfil lingüístico de los participantes, de modo que aquellos que han estudiado y/o utilizan habitualmente una lengua propia además del castellano (en concreto, el catalán) muestran actitudes más favorables hacia este tipo de enseñanza que quienes tienen un perfil monolingüe castellano, que muestran actitudes neutras. Este hecho corrobora la tendencia encontrada en investigaciones como la de Saladich (2012), que apunta a que las personas que hablan varias lenguas muestran una mayor sensibilidad hacia la diversidad lingüística que las personas monolingües como consecuencia del desarrollo de una conciencia pluricultural y plurilingüe. En cualquier caso, la situación encontrada también podría entenderse como una evidencia de la presencia de actitudes de lealtad lingüística (Niculescu, 1996) entre los hablantes de lenguas minoritarias, entendiendo que estos sujetos encuentran en el marco de la educación multilingüe el modo de preservar su herencia lingüística.

Tampoco debemos obviar las diferencias encontradas en función del curso en el que se encuentran los maestros en formación pues, a tenor de los resultados obtenidos, parece que el paso por los estudios del Grado en Magisterio en Educación Primaria podría redundar en una mejora significativa en las actitudes hacia la educación multilingüe, encontrándose actitudes neutrales (aunque con tendencia favorable) entre el alumnado de primer curso y favorables en el caso del alumnado de cuarto.

También nos parece oportuno subrayar las diferencias encontradas según el sexo de los participantes en lo que respecta a las actitudes hacia la educación multilingüe, pero también hacia el francés, de modo que las mujeres presentan valoraciones más positivas que los hombres en los dos casos. En este sentido, y en coherencia con la literatura previa, resulta complejo llegar a una conclusión sólida ya que, tal y como apunta tras un profundo análisis teórico Lasagabaster (2003), la falta de sistematicidad de las investigaciones centradas en el análisis de la relación entre las actitudes lingüísticas y la variable sexo no ha permitido llegar 
a conclusiones definitivas. En cualquier caso, el resultado encontrado en nuestro estudio es similar al hallado en investigaciones previas realizadas en el mismo territorio pero con cohortes más jóvenes (Campos ,2021).

Finalmente, cabe señalar que la actitud general de orientación favorable que encontramos entre los futuros maestros por lo que respecta a la educación multilingüe podría constatar la tendencia de este colectivo a valorar de forma positiva la diversidad y la comunicación intercultural, realidad que se ha evidenciado en estudios recientes como el de Pareja de Vicente, Leiva Olivencia y Matas Terrón (2020). El efecto de la variable curso, con una modificación de las actitudes desde neutras hasta favorables, podría constatar en este sentido el impacto positivo de la formación recibida en los estudios en Magisterio.

\section{Referencias}

Bradac, J., Cargile, A. Y Hallett, J. (2001). Language attitudes: retrospect, conspect and prospect. En W.P. Robinson y H. Giles (Eds.), The new Handbook of Language and Social Psychology (pp. 138-155). Wiley: Chichester.

Baquedano, P. (Coord.) (2019). Informe 2019 sobre la situación del Sistema Educativo en Aragón. Zaragoza: Consejo Escolar de Aragón.

Campos, I.O. (2013). Un acercamiento a las actitudes lingüísticas del alumnado del Grado en Maestro de Educación Primaria de los campus de Huesca y Zaragoza. Estudio piloto. Alazet, 25, 31-56.

Campos, I.O. (2014). ¿L'aragonés en a escuela? Estudio de las actitudes lingüísticas del futuro profesorado aragonés de Educación Primaria. En Lozano, Ch. y Sorolla, N. (Coords.), Actas de las II Jornadas Aragonesas de Sociología (pp. 8-29). Zaragoza: Prensas Universitarias de Zaragoza, Gara d’Edizions e Institución Fernando el Católico.

Campos, I.O. (2015). Actitudes hacia el catalán del futuro profesorado aragonés de Educación Primaria. Sintagma, 27, 43-59.

Campos, I.O. (2021). Las actitudes lingüísticas en los centros de Educación Secundaria del Alto Aragón. Evidencias para la actualización del estado de la cuestión. Zaragoza: Prensas de la Universidad de Zaragoza.

Herrera, J. y Pozas, M.N. (2020). Enseñar en la sociedad de la información y el conocimiento. Creencias acerca de la formación inicial del profesorado de lenguas extranjeras inglés y francés- en Educación Infantil. Revista Qurriculum, 22, 129-150. DOI : https://doi.org/10.25145/j.qurricul.2020.33.07

Marín, R., Alzás, T. y Villares, S. (2020). Creencias de los alumnos del grado de educación sobre el proceso de aprendizaje de la lengua inglesa. Campo Abierto, 39(1), 71-83. DOI: 10.17398/0213-9529.39.1.71

Consejo de Europa (2001). Common European Framework for Languages: Learning, teaching, assessment. Estrasburgo: Council for Cultural Cooperation-Education Comittee, Language Policy Division.

Gardner, R. (1985). Social Psychology and Second Language Learning: the role of attitudes and motivation. Londres: Arnold.

González Riaño, X.A. y Armesto, X. (2004). Les llengües n’Asturies: usu y valoración de la so importancia educativa- Estudiu empíricu fechu col alumnáu de Maxisteriu de la Universidá d'Uviéu. Oviedo-Uviéu: Academia de la Llingua Asturiana. 
González Riaño, X.A. y Huguet, Á. (2002). Estudio comparado de las actitudes lingüísticas de los escolares en contextos de minorización linguistica. Estudios de Sociolingüística, 3, 249-276.

Guadamillas, V. y Alcaraz, G. (2017). Legislación en enseñanza bilingüe: análisis en el marco de Educación Primaria en España. Multiárea: Revista de didáctica, 9, 82-103.

Huguet, Á. y González Riaño, X.A. (2004). Actitudes lingüísticas, lengua familiar y enseñanza de la lengua minoritaria. Barcelona: Horsori.

Janés, J. (2006). Las actitudes hacia las lenguas y el aprendizaje lingüístico. Revista Interuniversitaria de Formación del Profesorado, 20(2), 117-132.

Lambert, W., Hodgson, R., Gardner, R., y Fillenbaum, S. (1960). Evaluational reactions to spoken language. Journal of Abnormal and Social Psychology, 60, 44-51.

Lasagabaster, D. (2003). Trilingüismo en la enseñanza. Lleida: Milenio.

Lasagabaster, D. (2004). Un análisis de las actitudes hacia el inglés como lengua extranjera por medio de grupos de discusión. Estudios de Lingüísitca Aplicada, 40, 91-113.

Lasagabaster, D. y Huguet, Á. (Eds.) (2007). Multilingualism in European bilingual contexts. Language use and attitudes. Clevedon: Multilingual Matters.

Liberal, M. (2013). Casi dos décadas de enseñanza bilingüe en Aragón. Fórum Aragón, 10, 6-9. López, J.I. (Coord.) (2014). El aragonés en el siglo XXI. Zaragoza: Fundación Gaspar Torrente.

Ministerio de Educación y Formación Profesional (2019). Datos y cifras. Curso escolar 2018/2019. Madrid: Secretaría General Técnica de la Subdirección General de Atención al Ciudadano.

Morales, C., Arrimadas, I., Ramírez, E., López, A. y Ocaña, L. (2001). La enseñanza de lenguas extranjeras en España. Madrid: Centro de Investigación y Documentación Educativa.

Moreno Cabrera, J.C. (2008). El nacionalismo lingüístico: una ideologia destructiva. Barcelona: Península.

Moreno Fernández, F. (1998). Principios de Sociolingüística y Sociología del Lenguaje. Barcelona: Ariel.

Niculescu, A. (1996). Loyauté linguistique. En H. Goebl, P.H. Nelde, Z. Stary y W. Wolck (Coords.), Contacts Linguistics (pp. 715-720). Berlin: Mouton de Gruyter.

Ó Rigain, P. (2008). Language attitudes and minority languages. En J. Cenoz y N. Hornberger (Eds.), Encyclopedia of Language and Education. Volume 6: Knowlegge about Language (pp. 329-341). Londres: Springer.

Pareja de Vicente, D. Leiva Olivencia, J.J. y Matas Terrón, A. (2020). Percepciones sobre diversidad cultural y comunicación intercultural de futuros maestros. Revista Electrónica Interuniversitaria de Formación del Profesorado, 23(1), 75-87. DOI: https://doi.org/10.6018/reifop.403331

Saladich, M.J. (2012). Representacions i acctituds del professorat de llengües de secundària envers la diversitat lingüística i cultural. REIRE, 5(2), 22-41.

Sharp, D., Thomas, B., Price, E., Francis, G. y Davis, I. (1973). Attitudes to Welsh and English in the Schools of Wales. Basingstoke/Cardiff: McMillan y University of Wales Press.

Siguán, M. (2005). La Europa de las lenguas. Madrid: Alianza. 